\title{
Enfermería en salud ocupacional: una mirada a los instrumentos utilizados en investigación a nivel internacional ${ }^{1}$
}

\author{
Juan Domingo Reyes Luna ${ }^{2}$, Sandra Valenzuela Suazo ${ }^{3}$, Varinia Rodríguez Campo ${ }^{4}$
}

Institución: Universidad de Concepción Chile

\section{RESUMEN}

El objetivo de este artículo fue identificar y caracterizar los instrumentos utilizados en salud laboral a nivel internacional, con la finalidad de obtener información relevante desde los resultados obtenidos. Se realizó una revisión integrativa de la literatura científica en 6 bases de datos, según método analítico SALSA, como una forma de identificar los instrumentos de evaluación en salud laboral utilizados en el periodo 2012 - 2017. De la revisión se obtuvo 61 instrumentos utilizados en investigación en salud laboral, en el ámbito internacional, de la triangulación de ellos se logró como resultado cinco dimensiones: psicosocial - capacidad y condiciones de trabajo - factores de riesgo y detección de la enfermedad - autoestima, empoderamiento, motivación - calidad de vida. Debido a la gran cantidad de instrumentos encontrados, la contribución de este artículo es recopilar, caracterizar y agrupar la información en dimensiones, según características y objetos de estudio, de tal forma de orientar a los profesionales de Enfermería que decidan investigar en esta temática, un área en la que se enfrenta grandes retos para la profesión: por un lado, estimular la generación de evidencia científica en el área de enfermería en salud ocupacional, desde la visión disciplinar integradora del cuidado y por otro, la posibilidad de validar estos instrumentos y evaluar así su aplicabilidad en población trabajadora específica.

Palabras clave: Instrumentos; Investigación-en-Enfermería; Salud-Laboral.

DOI 10.15517/revenf.v0iNo. 37.34876

${ }^{1}$ Fecha de recepción: 15 de octubre del 2018

${ }^{2}$ Estudiante programa de Doctorado de Enfermería, Universidad de Concepción, Chile. Correo: jreyes1@,udec.cl

${ }^{3}$ Doctora en Enfermería, de Facultad de Enfermería, Universidad de Concepción, Chile. Correo: svalenzu@udec.cl

${ }^{4}$ Doctora en Enfermería, de Facultad de Enfermería, Universidad de Concepción, Chile. Correo: varirodriguez@udec.cl 


\title{
Nursing in occupational health: a look at the instruments used in research at the international level ${ }^{1}$
}

\author{
Juan Domingo Reyes Luna ${ }^{2}$, Sandra Valenzuela Suazo ${ }^{3}$, Varinia Rodríguez Campo ${ }^{4}$
}

Institution: University of Concepción Chile

\begin{abstract}
The objective of this article was identify and characterize the instruments used in occupational health internationally, with the purpose of collecting relevant information regarding dimensions according to the characteristics of the results obtained. An integrative revision of available scientific literatura was performed including 6 databases, as a means to identify the assessment instruments used in occupational health during the period 2012 - 2017. From the above, 61 instruments used in occupational health research were obtained, from the triangulation of which 5 dimensions were reached: psychosocial - working conditions and capability - risk factors and disease detection - self-esteem, empowerment, motivation - quality of life. Due to the great number of instruments found, the contribution of this article is the compilation, characterization and grouping of the information into dimensions according to characteristics and subjects of study, in such a way as to offer guidance to the professional nurse aiming to carry out research on this particular matter. Great challenges in this field are both to stimulate the curiosity towards research with a higher ambition for scientific evidence, and the research regarding workers on a day-to-day basis as, without a shred of doubt it is thus that we can make our work visible to society.
\end{abstract}

Key words: Instruments; Nursing-Research; Occupational-Health.

DOI 10.15517/revenf.v0iNo. 37.34876

${ }^{1}$ Date of receipt: October 15, 2018

Date of acceptance: March 11, 2018

${ }^{2}$ Student Doctoral Program in Nursing, University of Concepción, Chile. E-mail: jreyesl@udec.cl

${ }^{3}$ Doctor in Nursing, Faculty of Nursing, University of Concepción, Chile. E-mail: svalenzu@udec.cl

${ }^{4}$ Doctor in Nursing, Faculty of Nursing, University of Concepción, Chile. E-mail: varirodriguez@udec.cl 


\title{
Enfermagem em saúde do trabalho: um olhar para os instrumentos mais utilizados em investigação em âmbito internacional ${ }^{1}$
}

\author{
Juan Domingo Reyes Luna ${ }^{2}$, Sandra Valenzuela Suazo ${ }^{3}$, Varinia Rodríguez Campo ${ }^{4}$
}

Instituição: Universidade de Concepción Chile

\section{RESUMO}

O objetivo deste artigo foi identificar e caracterizar os instrumentos utilizados em saúde do trabalho no âmbito internacional, com a finalidade de obter informação relevante com vistas a estabelecer dimensões conforme características dos resultados obtidos. Metodologia: Realizou-se uma revisão integrativa da literatura científica, segundo o método analítico SALSA, com busca em 6 bases de dados, como uma forma de identificar os instrumentos de avaliação em saúde do trabalho utilizados no período 2012-2017. Foram obtidos 61 instrumentos utilizados em investigação em saúde do trabalho no âmbito internacional, de cuja triangulação obtiveram-se 5 dimensões: Psicossocial, Capacidade e Condições de Trabalho, Fatores de Risco e Detecção da Doença, Autoestima, Empoderamento e Motivação, Qualidade de Vida. Devido a grande quantidade de instrumentos encontrados, a contribuição desse artigo reside em compilar, caracterizar e agrupar a informação em dimensões segundo características e objetos de estudo, de tal forma a proporcionar uma orientação aos profissionais de Enfermagem que decidam investigar sobre a temática. Nesse sentido, tem-se grandes desafios para a profissão: por um lado, estimular a inquietação de investigar, contudo com maior ambição de evidência científica; por outro lado, investigar com base no cotidiano do trabalhador, uma vez que a partir desse olhar podemos visibilizar o nosso fazer na sociedade.

Palavras chaves: Instrumentos; Pesquisa-em-Enfermagem; Saúde-Ocupacional.

DOI 10.15517/revenf.v0iNo. 37.34876

${ }^{1}$ Data de recepção: 15 de outubro de 2018

Data de aceitação: 11 de março de 2018

2 Estudante do Programa de Doutorado em Enfermagem, Universidade de Concepción, Chile. Correio eletrônico: jreyesl@udec.cl

${ }^{3}$ Doutor em Enfermagem, Escola de Enfermagem, Universidade de Concepción, Chile. Correio eletrônico: svalenzu@udec.cl

${ }^{4}$ Doutor em Enfermagem, Escola de Enfermagem, Universidade de Concepción, Chile. Correio eletrônico: varirodriguez@udec.cl 


\section{INTRODUCCIÓN}

El trabajo es un derecho fundamental de las personas, a través del que sustentan su diario vivir y, por supuesto, satisfacen sus necesidades básicas y que abarca más allá de lo físico, ya que también es un medio con el que los individuos enfrentan a la sociedad, familia, e incluso su identidad ${ }^{1}$. En este contexto es que, desde el año 2004, la Organización Internacional del Trabajo (OIT) hace un llamado a la mayoría de los países del mundo a buscar estrategias que aseguren el bienestar de los trabajadores y propone dar solución a "temas especiales" como la generación de trabajo decente, especialmente en el Mercado Común del Sur (MERCOSUR), así como a elevar la productividad laboral, crecimiento y bienestar en el trabajo y abolir el trabajo infantil en América latina y el Caribe ${ }^{1}$.

$\mathrm{Al}$ anterior contexto se suma el informe de la Organización Mundial de la Salud (OMS) ${ }^{2}$ sobre la salud en el mundo, cuyo lema es "trabajando juntos por la salud", el cual comenta la necesidad urgente de que exista preocupación mundial por la crisis de la fuerza de trabajo en salud, sobre todo en los países más pobres donde la escasez es aún mayor en los trabajadores de la salud ${ }^{2}$, situación que fue debatida, posteriormente, en los foros de Kampala $^{3}$, Bangkok ${ }^{4}$, Recife ${ }^{5}$ : todas coinciden en que es necesario procurar la salud de los trabajadores en un entorno y sistema adecuados.

De lo expuesto, en medio de este ambiente no propicio para la fuerza laboral, en la última década ha crecido el interés por estudiar y abordar estas y otras temáticas de la salud de los trabajadores de lo que surgen, entonces, los indicadores de salud, en los que la persona informa sobre su salud: indicadores de resultados por los pacientes $^{6}$. Una de las formas de abordar esta problemática, es a través de la investigación en la que los estudios científicos representa una de las principales vías para que esta información sea divulgada y socializada a la comunidad científica; por tanto, el rigor metodológico en la investigación y los instrumentos de medición utilizados y validados para cumplir los objetivos propuestos son fundamentales puesto que, finalmente, pueden llegar a constituirse en un instrumento social que promueva la mejoría del cuidado de la salud de los ciudadanos ${ }^{7}$.

Respecto de lo anterior, la Enfermería cuyo eje central es el cuidado de los seres humanos, familia y comunidad, también se involucra con el cuidado de los trabajadores: en este caso, se llevó a cabo una revisión cuyo objetivo es identificar y caracterizar los instrumentos utilizados en salud ocupacional a nivel internacional, con el fin de obtener instrumentos válidos y confiables que aseguren el aporte de evidencias para ser aplicadas en el mundo laboral y, de esta manera, favorecer el cuidado de los trabajadores.

\section{MATERIALES Y MÉTODOS}

Previo a la búsqueda, es necesario plantear la pregunta de investigación, la cual cuestiona ¿qué instrumentos se utilizan en investigación en salud ocupacional y cuáles son las dimensiones y características de estas herramientas? Para conocer el estado del arte, se desarrolló una revisión integrativa que reúne la mejor evidencia disponible $^{8}$ de la literatura internacional y que permite sintetizar el conocimiento ${ }^{9}$, y los resultados de diversas investigaciones sobre un tema de manera sistemática y ordenada ${ }^{10}$, la cual genera actualización y profundización del tema. Para la búsqueda de información se utilizó el método analítico simple SALSA de revisión(Search, AppraisaL, Syntesis, Analysis) ${ }^{11}$, es decir, que los objetos de estudios deben proceder de una 
búsqueda (search), para ser evaluados (appraisaL), sintetizados de acuerdo con un esquema (syntesis) y, finalmente, los resultados deben ser analizados y presentados de forma coherente (analisys).

La estrategia de búsqueda de la información se llevó a cabo durante el periodo septiembre-diciembre del 2017: inició con una definición inicial de términos controlados y referenciados en los descriptores en ciencias de la salud ( DeCS) y MeSH (Medical Subject Headings) con el que se construyó ecuaciones de búsqueda para ser utilizadas en cada una de las bases de datos consultadas (tabla 1), tales como Medline (Pubmed), web of sciencie (WoS), Biblioteca Virtual en Salud (BVS), ScIELO (Scientific Electronic Library Online o Biblioteca Científica Electrónica en Línea), LILAC ( Literatura Latinoamericana de Información en Ciencias de la Salud); en cuanto a los descriptores en español se utilizó "salud ocupacional", "investigación", "enfermeria" "occupational, mientras que en inglés fueron "health", "research", "nursing” y "saúde ocupacional”, "investigação"," enfermagem”, en portugués.

Respecto de los criterios de inclusión, se consideró artículos de investigación cuantitativa; trabajadores adultos en el ámbito laboral; últimos cinco años; idiomas inglés, portugués y español. Los criterios de exclusión abarcaron estudios cualitativos, revisiones sistemáticas e integrativas; metaanálisis; menores de edad y/o estudiantes y literatura gris (folletos, tesis, cartas al editor, artículos de opinión).

Esta exhaustiva revisión permitió ubicar un amplio volumen de trabajos de investigación que, luego de la lectura de títulos y resúmenes y de la lectura crítica y comprensiva del texto completo, permitió seleccionar artículos relevantes para el fenómeno del estudio, a través de una pauta organizadora de datos que incluyó los siguientes ítems: autor, nombre del artículo, año, bases de datos, palabras claves, lugar de estudio, objetivos, metodología, instrumentos, resultados, conclusiones, datos que permiten construir tablas de resúmenes.

\section{RESULTADOS}

Luego de una rigurosa búsqueda de la que se obtuvo 11323 artículos, finalmente se seleccionó 45 que cumplían los requisitos de inclusión.

Tabla 1. Universidad de Concepción de Chile. Resultados de la búsqueda por base de datos, 2017.

\begin{tabular}{|c|c|c|c|c|c|c|}
\hline BASE DE DATOS & $\begin{array}{c}\text { FILTROS } \\
\text { BASE A TÉRMINOS }\end{array}$ & $\begin{array}{c}\text { EXCLUSIÓN } \\
\text { POR NO OBJETO } \\
\text { DE ESTUDIO } \\
\end{array}$ & $\begin{array}{l}\text { SELECCIÓN } \\
\text { DE TÍTULO } \\
\text { Y RESUMEN } \\
\end{array}$ & $\begin{array}{c}\text { DUPLICADOS } \\
\text { BASE DE DATOS }\end{array}$ & $\begin{array}{l}\text { EXCLUSION } \\
\text { POR NO OBJETO } \\
\text { DE ESTUDIO } \\
\end{array}$ & $\begin{array}{c}\text { LECTURA } \\
\text { TEXTO } \\
\text { COMPLETO } \\
\end{array}$ \\
\hline WOS & 144 artículos & 129 artículos & 15 artículos & 0 artículos & 6 artículos & 9 artículos \\
\hline MEDLINE & 1021 artículos & 924 artículos & 97 artículos & 2 artículos & 89 artículos & 6 artículos \\
\hline SCIELO & 53 artículos & 24 artículos & 29 artículos & 1 artículos & 22 artículos & 6 artículos \\
\hline BVS & 9556 artículos & 9527 artículos & 29 artículos & 2 artículos & 21 artículos & 6 artículos \\
\hline \multirow[t]{2}{*}{ LILACS } & 549 artículos & 383 artículos & 166 artículos & 2 artículos & 146 artículos & 18 artículos \\
\hline & 11323 & & & & & 45 artículos \\
\hline
\end{tabular}

Fuente: elaboración propia. 
En la exhaustiva revisión de la literatura, se encontró 61 instrumentos de medición de un total de 45 estudios seleccionados, los cuales fueron utilizados por los profesionales para investigar el tema de salud ocupacional.

Tabla 2. Universidad de Concepción de Chile. Resultados de los instrumentos incluidos en la revisión en el ámbito internacional, 2017.

\begin{tabular}{|c|c|c|c|c|}
\hline Nombre instrumento & Sigla & Característica & Autor & Nombre artículo/Revista \\
\hline $\begin{array}{l}\text { Escala de costo } \\
\text { humano en el trabajo }\end{array}$ & ECHT & $\begin{array}{l}\text { Evalúa la energía que el trabajador expresa, } \\
\text { la energía que le lleva trabajar } \\
\text { individualmente en la esfera cognitiva- } \\
\text { emocional - física. }\end{array}$ & $\begin{array}{l}\text { Méndez } \\
\text { ( 2007) }\end{array}$ & $\begin{array}{l}\text { Custo humano no trabalho em } \\
\text { enfermagem em um serviço de } \\
\text { hemodiálise }^{12} \\
\text { / Revista Baiana de Enfermagem, } \\
\text { Salvador }\end{array}$ \\
\hline $\begin{array}{l}\text { Cuestionario de estrés } \\
\text { de la conciencia }\end{array}$ & SCQ & $\begin{array}{l}\text { Mide estrés de conciencia: la parte A mide la } \\
\text { frecuencia de las situaciones estresante y la } \\
\mathrm{B} \text {, la cantidad de estrés de conciencia que se } \\
\text { generó a partir de la situación estresante }\end{array}$ & $\begin{array}{l}\text { Glasberg } \\
\text { et al. (2007) }\end{array}$ & $\begin{array}{l}\text { Longitudinal relationships between } \\
\text { stress of conscience and concepts of } \\
\text { importance }{ }^{13} \\
\text { /Journal Nursing Ethics }\end{array}$ \\
\hline $\begin{array}{l}\text { Cuestionario de } \\
\text { percepción de la } \\
\text { conciencia }\end{array}$ & PCQ & $\begin{array}{l}\text { Se evalúa la percepción de conciencia, su } \\
\text { origen, función y significancia. }\end{array}$ & $\begin{array}{l}\text { Dahlqvist } \\
\text { et al (2007) }\end{array}$ & $\begin{array}{l}\text { Artículo } \\
\text { /Journal Nursing Ethics }\end{array}$ \\
\hline $\begin{array}{l}\text { Cuestionario } \\
\text { del clima de cuidado } \\
\text { centrado en la persona }\end{array}$ & PCCQ & $\begin{array}{l}\text { Diseñado para medir en qué medida el } \\
\text { personal de salud evalúa en clima de } \\
\text { cuidado centrado en la persona. }\end{array}$ & $\begin{array}{l}\text { Edvardsson D; } \\
\text { Sandman PO; } \\
\text { Rasmussen B. } \\
(2009)\end{array}$ & $\begin{array}{l}\text { Artículo }^{13} \\
\text { /Journal Nursing Ethics }\end{array}$ \\
\hline $\begin{array}{l}\text { Cuestionario } \\
\text { de apoyo } \\
\text { social }\end{array}$ & SOCIS & $\begin{array}{l}\text { Centrado en el entorno del trabajo, sobre el } \\
\text { apoyo de un gerente - compañero de } \\
\text { trabajo-familia, amigos. }\end{array}$ & $\begin{array}{l}\text { Dalner M. } \\
\text { et al. } \\
(2000)\end{array}$ & $\begin{array}{l}\text { Artículo }^{13} \\
\text { /Journal Nursing Ethics }\end{array}$ \\
\hline $\begin{array}{l}\text { Cuestionario } \\
\text { de } \\
\text { motivación } \\
\text { para el trabajo }\end{array}$ & CMT & $\begin{array}{l}\text { Mide factores motivacionales: ingreso, } \\
\text { recompensa, participación, autoridad, } \\
\text { aprecio, seguridad, oportunidad, promoción, } \\
\text { trabajo equipo. }\end{array}$ & $\begin{array}{l}\text { Korkmaz } \\
(2008)\end{array}$ & $\begin{array}{l}\text { Nurses' perceptions of motivational } \\
\text { factors: a case study in a Turkish } \\
\text { university hospital }{ }^{14} \\
\text { /Journal of nursing managenement }\end{array}$ \\
\hline $\begin{array}{l}\text { Cuestionario de } \\
\text { satisfacción laboral }\end{array}$ & $\mathrm{S} 10 / 12$ & $\begin{array}{l}\text { Medir satisfacción laboral describiendo tres } \\
\text { factores: satisfacción con la supervisión, } \\
\text { satisfacción con el ambiente físico, } \\
\text { satisfacción con las prestaciones recibidas. }\end{array}$ & $\begin{array}{l}\text { Melia y Peiro } \\
(1998)\end{array}$ & $\begin{array}{l}\text { Psychosocial study about the } \\
\text { consequences of work in hospital } \\
\text { nurses as human resource } \\
\text { management } \\
\text { /Revista Latinoamérica de Enfermagem }\end{array}$ \\
\hline $\begin{array}{l}\text { Cuestionario } \\
\text { salud general de } \\
\text { Goldberg }\end{array}$ & GHQ-28 & $\begin{array}{l}\text { Sugerido para valoración de salud mental, } \\
\text { cuidadores, para detectar problemas de } \\
\text { nueva aparición. }\end{array}$ & $\begin{array}{l}\text { Goldberg } \\
\text { (1979) }\end{array}$ & $\begin{array}{l}\text { Artículo }{ }^{15} \\
\text { /Revista Latinoamérica Enfermagem }\end{array}$ \\
\hline $\begin{array}{l}\text { Cuestionario de salud } \\
\text { y rendimiento del } \\
\text { trabajo }\end{array}$ & HPQ & $\begin{array}{l}\text { Autoinforme diseñado para estimar los } \\
\text { costos laborales de salud en términos de } \\
\text { reducción de rendimiento en el trabajo, } \\
\text { ausencia de enfermedad o accidentes. }\end{array}$ & OMS & $\begin{array}{l}\text { Adaptação transcultural e validação do } \\
\text { World Health Organization Health and } \\
\text { Work Performance Questionnaire para } \\
\text { enfermeiros brasileiros }{ }^{16} \\
\text { / Revista da Escola Enfermagem da } \\
\text { USP }\end{array}$ \\
\hline $\begin{array}{l}\text { Cuestionario de } \\
\text { relación consumo de } \\
\text { ansiolítico y trabajo }\end{array}$ & QRCAT & $\begin{array}{l}\text { Identifica desorden relacionado con el } \\
\text { consumo. }\end{array}$ & $\begin{array}{l}\text { Barbosa de Oliveira } \\
\text { E. }(2014)\end{array}$ & $\begin{array}{l}\text { Estresse ocupacional e consumo de } \\
\text { ansiolíticos por trabalhadores de } \\
\text { enfermagem }^{17} \\
\text { / Revista enfermagem UERJ }\end{array}$ \\
\hline
\end{tabular}




\begin{tabular}{|c|c|c|c|c|}
\hline $\begin{array}{l}\text { Cuestionario de } \\
\text { condiciones de trabajo } \\
\text { (The Working } \\
\text { Conditions Survey) }\end{array}$ & QCT & $\begin{array}{l}\text { Herramienta para evaluar dimensión } \\
\text { psicosocial, subjetiva y percibida de las } \\
\text { condiciones de trabajo. }\end{array}$ & $\begin{array}{l}\text { Blanch } \\
(2005)\end{array}$ & $\begin{array}{l}\text { Condiciones de trabajo de las } \\
\text { enfermeras y las formas de contratación } \\
\text { en Medellín, } \\
\text { Colombia }{ }^{18} \\
\text { /Revista Universidad y Salud }\end{array}$ \\
\hline $\begin{array}{l}\text { Cuestionario de } \\
\text { limitaciones en trabajo } \\
\text { para la evaluación del } \\
\text { presentismo }\end{array}$ & WLQ & $\begin{array}{l}\text { Mide el efecto de la salud en la } \\
\text { productividad organizacional. }\end{array}$ & $\begin{array}{l}\text { Lerner et al. } \\
(2003) ; \text { Ver-sión } \\
\text { Brasileña } \\
\text { Soárez et al. (2007) }\end{array}$ & $\begin{array}{l}\text { Estresse, coping e presenteísmo em } \\
\text { enfermeiros que assistem pacientes } \\
\text { críticos e potencialmente críticos } 19 \\
\text { / Revista da Escola Enfermagen da USP }\end{array}$ \\
\hline $\begin{array}{l}\text { Cuestionario relación } \\
\text { consumo alcohol con } \\
\text { el trabajo }\end{array}$ & QRCAT & $\begin{array}{l}\text { Identifica desórdenes relacionados con el } \\
\text { consumo de alcohol y el trabajo. }\end{array}$ & $\begin{array}{l}\text { Barbosa de Oliveira } \\
(2013)\end{array}$ & $\begin{array}{l}\text { Padrões de uso de álcool por } \\
\text { trabalhadores de enfermagem e a } \\
\text { associação com o trabalho }{ }^{20} \\
\text { / Revista enfermagem UERJ }\end{array}$ \\
\hline $\begin{array}{l}\text { Cuestionario } \\
\text { psicosocial de } \\
\text { de Copenhague }\end{array}$ & CoPsoQ & $\begin{array}{l}\text { Identificar y medir la exposición a factores } \\
\text { de riesgo para la salud de naturaleza } \\
\text { psicosocial en el trabajo. }\end{array}$ & $\begin{array}{l}\text { Instituto Nacional } \\
\text { de Salud Laboral de } \\
\text { Dinamarca, (2000) }\end{array}$ & $\begin{array}{l}\text { Work stressors and resources in a Swiss } \\
\text { orthopaedic clinic and their relationship } \\
\text { with } \\
\text { employees' health and the patient } \\
\text { orientation } \\
\text { of employees }{ }^{21} \\
/ \text { The european journal of medicine } \\
\text { sciencies }\end{array}$ \\
\hline $\begin{array}{l}\text { Cuestionario } \\
\text { Front Roja }\end{array}$ & CFRS & $\begin{array}{l}\text { Cuestionario de salud laboral: satisfacción } \\
\text { por el trabajo/tensión relacionada con el } \\
\text { trabajo/competencia profesional/presión en } \\
\text { el trabajo/promoción. }\end{array}$ & $\begin{array}{l}\text { Aranz J. } \\
(1988)\end{array}$ & 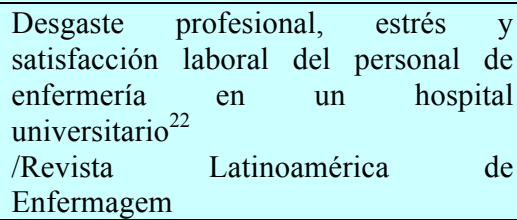 \\
\hline $\begin{array}{l}\text { Cuestionario } \\
\text { de Boix y Vogel }\end{array}$ & - & $\begin{array}{l}\text { Instrumento diseñado para evaluar las } \\
\text { condiciones de riesgo en el medio ambiente } \\
\text { del trabajo. }\end{array}$ & $\begin{array}{l}\text { Boix P. } \\
\text { Vogel L, } \\
(1997)\end{array}$ & $\begin{array}{l}\text { Riscos ocupacionais e problemas de } \\
\text { saúde percebidos por trabalhadores de } \\
\text { enfermagem em unidade hospitalar }{ }^{23} \\
\text { /Revista Ciencia y Enfermeria }\end{array}$ \\
\hline $\begin{array}{l}\text { Cuestionario } \\
\text { respiratorio } \\
\text { Saint George }\end{array}$ & SGRQ & $\begin{array}{l}\text { Evalúa la relación calidad de vida y } \\
\text { enfermedad pulmonar. }\end{array}$ & $\begin{array}{l}\text { Versión española } \\
\text { Ferrer, M. } \\
\text { et al (1996) }\end{array}$ & $\begin{array}{l}\text { Telemetric Detection of Chronic } \\
\text { Obstructive Pulmonary Disease and } \\
\text { Investigation of Quality of Life for } \\
\text { People Working in Shipbuilding } \\
\text { Industry }{ }^{24} \\
\text { / Revista Acta Informática Médica }\end{array}$ \\
\hline $\begin{array}{l}\text { Cuestionario } \\
\text { desequilibrio esfuerzo- } \\
\text { recompensa }\end{array}$ & C-ERI & $\begin{array}{l}\text { Aporta información de características del } \\
\text { trabajador como el esfuerzo que debe } \\
\text { realizar para completar sus tareas, la } \\
\text { recompensa que obtiene y el nivel } \\
\text { implicación con la empresa. }\end{array}$ & $\begin{array}{l}\text { Siegrist, J. } \\
\text { (1996) }\end{array}$ & $\begin{array}{l}\text { Mental health and poor recovery in } \\
\text { female } \\
\text { nursing workers: a contribution to the } \\
\text { study of gender inequities }{ }^{25} \\
\text { /BMC Psiquiatric }\end{array}$ \\
\hline 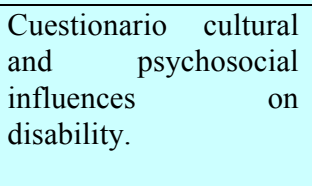 & CUPID & $\begin{array}{l}\text { Cuestionario investigación internacional } \\
\text { sobre las influencias físicas, culturales y } \\
\text { psicosociales en los síntomas músculo- } \\
\text { esqueléticos en incapacidad. }\end{array}$ & $\begin{array}{l}\text { Coggon D. } \\
\text { ( 2005) } \\
\text { Adaptación Brasil } \\
\text { Lepos A. (2010) }\end{array}$ & $\begin{array}{l}\text { Assessment of potential risk factors for } \\
\text { new onset disabling low back pain in } \\
\text { Japanese workers: findings from the } \\
\text { cupid study }{ }^{26} \\
\text { / BMC Musculoeskeletal disorders }\end{array}$ \\
\hline $\begin{array}{l}\text { Orebro } \\
\text { Musculoskeletal Pain } \\
\text { Questionnaire }\end{array}$ & OMPQ & $\begin{array}{l}\text { Predictivo: identificar pacientes con riesgo } \\
\text { de desarrollar problemas persistentes de } \\
\text { dolor de espalda en los últimos meses. }\end{array}$ & $\begin{array}{l}\text { Linton y Boersma } \\
(2003)\end{array}$ & $\begin{array}{l}\text { Artículo }^{26} \\
\text { /BMC Musculoeskeletal disorders }\end{array}$ \\
\hline $\begin{array}{l}\text { Cuestionario nórdico } \\
\text { estandarizado lesiones } \\
\text { bajas de la espalda }\end{array}$ & NSQ & $\begin{array}{l}\text { Detectar síntomas iniciales músculo- } \\
\text { esqueléticos en nueve regiones corporales: } \\
\text { cuello, hombros, parte superior espalda, } \\
\text { codos, zona lumbar, muñeca/manos, } \\
\text { caderas/muslos, rodillas y tobillos/pies. }\end{array}$ & $\begin{array}{l}\text { Kourinka I. et al. } \\
\text { (1987) }\end{array}$ & $\begin{array}{l}\text { Low back pain among school teachers } \\
\text { in Botswana, prevalence and risk } \\
\text { factors }{ }^{27} \\
\text { / BMC Musculoeskeletal disorders }\end{array}$ \\
\hline
\end{tabular}




\begin{tabular}{|c|c|c|c|c|}
\hline $\begin{array}{l}\text { Cuestionario } r \text { de } \\
\text { contenido del trabajo } \\
\text { (Job } \quad \text { Content } \\
\text { Questionnaire ) }\end{array}$ & JCQ & Identifica factores de riesgo psicosocial. & $\begin{array}{l}\text { Versión } 1.5 . \\
\text { Karasek, Pieper y } \\
\text { Schwartz, (1993) }\end{array}$ & $\begin{array}{l}\text { Artículo } \\
\text { / BMC Musculoeskeletal disorders }\end{array}$ \\
\hline $\begin{array}{l}\text { Cuestionario } \\
\text { satisfacción laboral }\end{array}$ & $\mathrm{S} 10 / 12$ & Mide satisfacción laboral. & $\begin{array}{l}\text { Melia J.L; } \\
\text { Peiró J.M. (1998) }\end{array}$ & $\begin{array}{l}\text { Artículo }^{15} \\
\text { /Revista Latinoamérica de Enfermagem }\end{array}$ \\
\hline $\begin{array}{l}\text { Cuestionario: medir la } \\
\text { calidad de vida }\end{array}$ & SF-36V2 & $\begin{array}{l}\text { Mide calidad de vida con la salud o salud } \\
\text { percibida. }\end{array}$ & Alonso J. (1995) & $\begin{array}{l}\text { Health-related quality of life and } \\
\text { associated factors of frontline railway } \\
\text { workers: A cross-sectional survey in } \\
\text { the Ankang Area, Shaanxi Province, } \\
\text { China }{ }^{28} \\
\text { /Journal of environment Research and } \\
\text { Public Health }\end{array}$ \\
\hline $\begin{array}{l}\text { Cuestionario: estrés } \\
\text { laboral } \\
\text { genérico }\end{array}$ & GJSQ & $\begin{array}{l}\text { Cuestionario que asocia el estrés en el } \\
\text { trabajo y la percepción de salud de los } \\
\text { trabajadores. }\end{array}$ & Nakata et al (2008) & $\begin{array}{l}\text { Work-family conflict as a mediator } \\
\text { between occupational stress and } \\
\text { psychological health among mental } \\
\text { health nurses in Japan }{ }^{29} \\
\text { /Neuropsychiatric disease and treatment }\end{array}$ \\
\hline $\begin{array}{l}\text { Job } \\
\text { content questionary }\end{array}$ & JCQ & $\begin{array}{l}\text { Evalúa factores de estrés laboral y el trabajo } \\
\text { relacionado con el ambiente. }\end{array}$ & $\begin{array}{l}\text { Versión china } \\
\text { Li J. (2004) }\end{array}$ & $\begin{array}{l}\text { Factors associated with burnout among } \\
\text { Chinese hospital doctors: a cross- } \\
\text { sectional study } \\
\text { / BMC Psiquiatric } \\
\text {-BMC Public Health } \\
\text {-Revista Latinoamérica Enfermagen }\end{array}$ \\
\hline $\begin{array}{l}\text { Escala indicadores de } \\
\text { placer y sufrimiento en } \\
\text { el trabajo }\end{array}$ & PSIWS & $\begin{array}{l}\text { Evalúa cuatro factores: dos experiencias de } \\
\text { placer y dos experiencias de sufrimientos. }\end{array}$ & $\begin{array}{l}\text { Validado en Brasil } \\
\text { Mendes AM; } \\
(2007)\end{array}$ & $\begin{array}{l}\text { Pleasure-suffering indicators of nursing } \\
\text { work in a hemodialysis nursing } \\
\text { service } \\
\text { / Revista da Escola Enfermagem da } \\
\text { USP }\end{array}$ \\
\hline $\begin{array}{l}\text { Escala de } \\
\text { afrontamiento } \\
\text { ocupacional }\end{array}$ & $\mathrm{ECO}$ & $\begin{array}{l}\text { Evalúa tres medidas de comportamiento de } \\
\text { afrontamiento relacionadas con el estrés } \\
\text { laboral: soporte social, agotamiento } \\
\text { emocional, sobrecarga de trabajo. }\end{array}$ & Latack (1986) & $\begin{array}{l}\text { Artículo } \\
/ \text { Revista da Escola Enfermagem da } \\
\text { USP }\end{array}$ \\
\hline $\begin{array}{l}\text { Escala de estrés } \\
\text { de Enfermeria }\end{array}$ & NSS & $\begin{array}{l}\text { Mide estresores laborales en el personal de } \\
\text { Enfermeria. }\end{array}$ & $\begin{array}{l}\text { Gray-toft, } \\
(1981)\end{array}$ & $\begin{array}{l}\text { Artículo }{ }^{19} \\
\text { / Revista Latinoamérica de } \\
\text { Enfermagem }\end{array}$ \\
\hline $\begin{array}{l}\text { Escala de satisfacción } \\
\text { laboral }\end{array}$ & $\mathrm{RN}$ & $\begin{array}{l}\text { Mide entorno de trabajo profesional, } \\
\text { autonomía, valor del trabajo, relaciones } \\
\text { profesionales, promulgación roles y } \\
\text { beneficios. }\end{array}$ & $\begin{array}{l}\text { Whitley y Putzier } \\
\text { (1994) }\end{array}$ & $\begin{array}{l}\text { Psychological empowerment and job } \\
\text { satisfaction between Baby Boomer and } \\
\text { Generation X nurses } \\
\text { /Journal of Nursing Management }\end{array}$ \\
\hline $\begin{array}{l}\text { Escala de síntomas } \\
\text { psicosomático } \\
\text { asociado al estrés }\end{array}$ & - & $\begin{array}{l}\text { Mide síntomas psicosomáticos asociados } \\
\text { con el estrés en el ambiente de trabajo. }\end{array}$ & $\begin{array}{l}\text { Golembiewski, } \\
\text { Munzenrider y } \\
\text { Carter (1983) } \\
\end{array}$ & $\begin{array}{l}\text { Artículo }{ }^{15} \\
/ \text { Revista Lat. Enfermagem }\end{array}$ \\
\hline $\begin{array}{l}\text { Escala } \\
\text { modificada } \\
\text { autoeficacia } \\
\text { del comportamiento }\end{array}$ & EEC-M & $\begin{array}{l}\text { Mide la autoeficacia en las muestras de } \\
\text { estudios. }\end{array}$ & $\begin{array}{l}\text { Hasting RP y } \\
\text { Brown T. } \\
(2002)\end{array}$ & $\begin{array}{l}\text { Artículo }^{15} \\
\text { /Journal of Clinical Nursing }\end{array}$ \\
\hline $\begin{array}{l}\text { Escala de bienestar } \\
\text { psicológico }\end{array}$ & - & $\begin{array}{l}\text { Evalúa dimensiones: autoaceptación, } \\
\text { relaciones positivas, dominio entorno, } \\
\text { autonomía, propósito de la vida, crecimiento } \\
\text { personal. }\end{array}$ & Ryff (1989) & $\begin{array}{l}\text { Artículo }^{15} \\
\text { /Revista Lat. Enfermagem }\end{array}$ \\
\hline $\begin{array}{l}\text { Escala de observación } \\
\text { de agresiones al } \\
\text { personal }\end{array}$ & SOAS-R & $\begin{array}{l}\text { Instrumento útil para la investigación, el } \\
\text { registro de incidentes y la gestión de } \\
\text { incidentes en la atención de emergencia. }\end{array}$ & $\begin{array}{l}\text { Palmstierna and } \\
\text { Wistedt (1987) }\end{array}$ & $\begin{array}{l}\text { Artículo }{ }^{15} \\
\text { /Journal of Clinical Nursing }\end{array}$ \\
\hline
\end{tabular}




\begin{tabular}{|c|c|c|c|c|}
\hline $\begin{array}{l}\text { Escala } \\
\text { Abreviada para medir } \\
\text { la calidad de } \\
\text { vida }\end{array}$ & $\begin{array}{l}\text { WHOQOL } \\
\text { BREF }\end{array}$ & $\begin{array}{l}\text { Proporciona un perfil de la calidad de vida } \\
\text { que la persona percibe: salud física, salud } \\
\text { psicológica, relaciones sociales, ambiente. }\end{array}$ & $\begin{array}{l}\text { Skevington } \\
\text { Sm et Al. } \\
(2004)\end{array}$ & $\begin{array}{l}\text { Association between sleep quality and } \\
\text { quality of life in nursing professionals } \\
\text { working rotating shifts } \\
\text { /Revista de Salud Pública }\end{array}$ \\
\hline $\begin{array}{l}\text { Escala del índice de } \\
\text { capacidad para el } \\
\text { trabajo }\end{array}$ & ICT & $\begin{array}{l}\text { Evalúa el rendimiento actual del trabajador y } \\
\text { estima las condiciones individuales. }\end{array}$ & $\begin{array}{l}\text { Llmarinen J. y } \\
\text { Tuomi K. } \\
(1991)\end{array}$ & 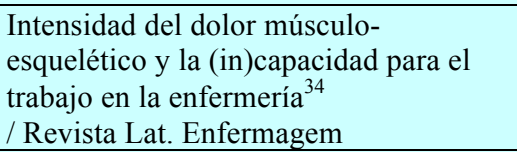 \\
\hline $\begin{array}{l}\text { Escala de self- report } \\
\text { Questionnaire- } 20\end{array}$ & SRQ-20 & $\begin{array}{l}\text { Evalúa trastornos mentales comunes en los } \\
\text { países en desarrollo. }\end{array}$ & $\begin{array}{l}\text { Mari JJ. y Williams } \\
\text { P. } \\
\text { (1986) }\end{array}$ & $\begin{array}{l}\text { Disturbios psíquicos menores en } \\
\text { enfermeros docentes universitarios } 35 \\
\text { / Revista Lat. Enfermagem } \\
\text { - Revista enfermagem UERJ }\end{array}$ \\
\hline $\begin{array}{l}\text { Escala de autoestima } \\
\text { de Rosemberg }\end{array}$ & ERA & $\begin{array}{l}\text { Evaluar el sentimiento de satisfacción que la } \\
\text { persona tiene de sí . }\end{array}$ & $\begin{array}{l}\text { Rosemberg } \\
(1965)\end{array}$ & $\begin{array}{l}\text { Avaliação da autoestima em docentes } \\
\text { de enfermagem de universidades } \\
\text { pública e privada } \\
\text { / Revista Lat. Enfermagem }\end{array}$ \\
\hline $\begin{array}{l}\text { Escala de esfuerzo y } \\
\text { recompensa }\end{array}$ & ERI & Modelo para anticiparse al estrés laboral. & $\begin{array}{l}\text { Siegrist y Peter } \\
(1998)\end{array}$ & $\begin{array}{l}\text { Artículo }{ }^{25} \\
\text { / Revista Española de Salud Pública }\end{array}$ \\
\hline $\begin{array}{l}\text { Escala del trabajo y } \\
\text { riesgo de enfermedad }\end{array}$ & ITRA & $\begin{array}{l}\text { Evalúa riesgo de enfermedad en el contexto } \\
\text { del trabajo. }\end{array}$ & $\begin{array}{l}\text { Mendez AM. } \\
(2007)\end{array}$ & $\begin{array}{l}\text { Artículo }^{31} \\
/ \text { Revista da Escola Enfermagem da } \\
\text { USP }\end{array}$ \\
\hline $\begin{array}{l}\text { Escala subjetiva } \\
\text { de carga mental } \\
\text { en trabajo }\end{array}$ & ESCAM & $\begin{array}{l}\text { Evalúa cinco dimensiones a) información } \\
\text { que el trabajador utiliza en su puesto de } \\
\text { trabajo, b) demandas cognitivas, c) } \\
\text { organización temporal del trabajo, d) } \\
\text { características de la tarea y e) consecuencias } \\
\text { para la salud del trabajador. }\end{array}$ & $\begin{array}{l}\text { Rolo G. et al. } \\
(2009)\end{array}$ & $\begin{array}{l}\text { Validación de escala subjetiva de carga } \\
\text { mental de trabajo en funcionarios/as } \\
\text { universitarios } 37 \\
\text { / Revista Ciencia y Enfermería }\end{array}$ \\
\hline $\begin{array}{l}\text { Evaluación } \\
\text { riesgo } \\
\text { psicosocial }\end{array}$ & $\begin{array}{l}\text { Suseso- } \\
\text { istas } 21\end{array}$ & $\begin{array}{l}\text { Mide los riesgos psicosociales en el } \\
\text { ambiente del trabajo, la versión breve está } \\
\text { diseñada para ser utilizada en empresas o } \\
\text { grupos de menos de } 25 \text { trabajadores. }\end{array}$ & $\begin{array}{l}\text { Adaptado en } \\
\text { Chile por } \\
\text { Alvarado } \\
\text { et al. (2009) }\end{array}$ & $\begin{array}{l}\text { Artículo }^{37} \\
\text { / Revista Ciencia y Enfermería }\end{array}$ \\
\hline $\begin{array}{l}\text { Job Strees } \\
\text { scale }\end{array}$ & JSS & Mide el estrés en el trabajo. & $\begin{array}{l}\text { Short versión } \\
\text { portugués } \\
\text { Guimarães de Bello } \\
(2004)\end{array}$ & $\begin{array}{l}\text { Capacidad para el trabajo en } \\
\text { enfermería: relación con demandas } \\
\text { psicológicas y control sobre el trabajo }{ }^{38} \\
\text { /Revista Latinoamericana Enfermagem }\end{array}$ \\
\hline $\begin{array}{l}\text { Escala de } \\
\text { enfrentamiento } \\
\text { de problema }\end{array}$ & EMEP & $\begin{array}{l}\text { Conceptualiza el enfrentamiento con un } \\
\text { conjunto de respuestas para una situación } \\
\text { estresante. }\end{array}$ & Vitalino et al (1993) & $\begin{array}{l}\text { A relação entre estressores } \\
\text { ocupacionais e estratégias de } \\
\text { enfrentamento em profissionais de nivel } \\
\text { tecnico de enfermagem } \\
\text { / Revista Texto \& Contexto } \\
\text { Enfermagem }\end{array}$ \\
\hline $\begin{array}{l}\text { Escala de inestabilidad } \\
\text { enfermera -trabajo }\end{array}$ & $\begin{array}{l}\text { Nurse- } \\
\text { wis }\end{array}$ & $\begin{array}{l}\text { Instrumento predictivo para registrar } \\
\text { inminente licencia por enfermedad a largo } \\
\text { plazo }\end{array}$ & $\begin{array}{l}\text { Gilworth G. } \\
\text { et al (2007) }\end{array}$ & $\begin{array}{l}\text { Predictive values and other quality } \\
\text { criteria of the german version of the } \\
\text { Nurse-Work Instability Scale (Nurse- } \\
\text { WIS) - follow-up survey findings of a } \\
\text { prospective study of a cohort of } \\
\text { geriatric care workers }{ }^{40} \\
\text { / Journal of occupational Medicine and } \\
\text { Toxicology }\end{array}$ \\
\hline $\begin{array}{l}\text { Escala de ansiedad de } \\
\text { autoevaluación de } \\
\text { Zung }\end{array}$ & EAA & $\begin{array}{l}\text { Evalúa manifestaciones características de } \\
\text { ansiedad, como síntomas o signos. }\end{array}$ & $\begin{array}{l}\text { Zung W. } \\
(1965)\end{array}$ & $\begin{array}{l}\text { Artículo }{ }^{16} \\
\text { / BMC Psiquiatric }\end{array}$ \\
\hline
\end{tabular}




\begin{tabular}{|c|c|c|c|c|}
\hline $\begin{array}{l}\text { Escale work- family - } \\
\text { conflict }\end{array}$ & WFC & $\begin{array}{l}\text { Mide el contenido al que el trabajo tiene } \\
\text { influencia en la familia. }\end{array}$ & $\begin{array}{l}\text { Netemeyer R. } \\
\text { G; Boles J.S; } \\
\text { Mc Murrian R. } \\
(1996)\end{array}$ & $\begin{array}{l}\text { Perceived Organizational Support } \\
\text { Impacts on the Associations of Work- } \\
\text { Family Conflict or Family-Work } \\
\text { Conflict with Depressive Symptoms } \\
\text { among Chinese Doctors }{ }^{41} \\
\text { / Journal of environment Research and } \\
\text { Public Health }\end{array}$ \\
\hline $\begin{array}{l}\text { Escale Family Work } \\
\text { Conflict }\end{array}$ & FWC & $\begin{array}{l}\text { Mide el contenido al que la familia tiene } \\
\text { influencia en el trabajo. }\end{array}$ & $\begin{array}{l}\text { Netemeyer R.; } \\
\text { Boles J.S; Mc } \\
\text { Murrian R. (1996) }\end{array}$ & $\begin{array}{l}\text { Artículo } \\
\text { / Journal of environment Research and } \\
\text { Public Health }\end{array}$ \\
\hline $\begin{array}{l}\text { Escale impediments in } \\
\text { the interaction with } \\
\text { patients }\end{array}$ & - & $\begin{array}{l}\text { Mide impedimentos en la interacción con los } \\
\text { pacientes. }\end{array}$ & $\begin{array}{l}\text { Rieder K; Faedi J; } \\
\text { Reinhard E. (2012) }\end{array}$ & $\begin{array}{l}\text { Work stressors and resources in a Swiss } \\
\text { orthopaedic clinic and their relationship } \\
\text { with employees' health and the patient } \\
\text { orientation of employees }{ }^{21} \\
\text { / The european journal of medicine } \\
\text { sciencies }\end{array}$ \\
\hline $\begin{array}{l}\text { Escale barriers } \\
\text { to patient } \\
\text { oriented work }\end{array}$ & - & $\begin{array}{l}\text { Mide las barreras al trabajo orientado con } \\
\text { pacientes. }\end{array}$ & $\begin{array}{l}\text { Rieder K; Faedi J; } \\
\text { Reinhard E.(2012) }\end{array}$ & $\begin{array}{l}\text { Artículo }^{21} \\
\text { /The european journal of medicine } \\
\text { sciencies }\end{array}$ \\
\hline $\begin{array}{l}\text { Escale } \\
\text { Patient- } \\
\text { oriented } \\
\text { control }\end{array}$ & - & $\begin{array}{l}\text { Mide el margen de maniobras que los } \\
\text { empleados tienen que tomar en sus propias } \\
\text { decisiones para actuar de una manera } \\
\text { orientada al paciente. }\end{array}$ & $\begin{array}{l}\text { Dormann C. } \\
(2003)\end{array}$ & $\begin{array}{l}\text { Artículo } \\
/ \text { The european journal of medicine } \\
\text { sciencies }\end{array}$ \\
\hline $\begin{array}{l}\text { Escale } \\
\text { patient } \\
\text { orientation }\end{array}$ & - & $\begin{array}{l}\text { Mide el deseo del empleado de responder } \\
\text { individualmente a las preferencias o } \\
\text { necesidades del paciente. }\end{array}$ & $\begin{array}{l}\text { Dormann C. } \\
(2003)\end{array}$ & $\begin{array}{l}\text { Artículo } \\
/ \text { The european journal of medicine } \\
\text { sciencies }\end{array}$ \\
\hline $\begin{array}{l}\text { Stanford Presenteeism } \\
\text { Scale }\end{array}$ & SPS-6 & $\begin{array}{l}\text { Mide el impacto de la capacidad percibida } \\
\text { de un trabajador para concentrarse en tareas } \\
\text { laborales a pesar de distracciones de } \\
\text { problemas de salud y dolor. }\end{array}$ & $\begin{array}{l}\text { Koopman C. } \\
\text { et al. }(2010)\end{array}$ & $\begin{array}{l}\text { Artículo } \\
\text { /The european journal of medicine } \\
\text { sciencies }\end{array}$ \\
\hline $\begin{array}{l}\text { Leyman inventory } \\
\text { psychological } \\
\text { terrorization }\end{array}$ & LIPT & $\begin{array}{l}\text { Enumera acciones sobre la víctima de acoso } \\
\text { moral }\end{array}$ & Heinz L. (1990) & $\begin{array}{l}\text { Factores asociados al acoso moral en el } \\
\text { ambiente laboral del enfermero }{ }^{42} \\
\text { / Revista Latinoamérica de } \\
\text { Enfermagem }\end{array}$ \\
\hline $\begin{array}{l}\text { Inventario de la } \\
\text { evaluación de factores } \\
\text { estresores subjetivos }\end{array}$ & IEE & $\begin{array}{l}\text { Evalúa el estrés ocupacional en enfermeros } \\
\text { en el entorno laboral. }\end{array}$ & $\begin{array}{l}\text { Stacciarini J; } \\
\text { Bartholomeau } \\
\text { T. } \\
\text { (2000) }\end{array}$ & $\begin{array}{l}\text { O estresse do trabalhador de } \\
\text { enfermagem: estudo em diferentes } \\
\text { unidades de um hospital universitário }{ }^{43} \\
\text { / Revista enfermagem UERJ } \\
\text { - Revista da Escola Enfermagem da } \\
\text { USP }\end{array}$ \\
\hline $\begin{array}{l}\text { Inventario de Burnout } \\
\text { de Maslach }\end{array}$ & MBI & $\begin{array}{l}\text { Evalúa tres dimensiones: agotamiento } \\
\text { emocional-despersonalización- realización } \\
\text { personal. }\end{array}$ & $\begin{array}{l}\text { Maslach and } \\
\text { Jackson } \\
(1981)\end{array}$ & $\begin{array}{l}\text { Síndrome de Burnout en el equipo de } \\
\text { salud de una unidad de cuidados } \\
\text { intensivos, Concepción } \\
\text { / BMC Public Health } \\
\text {-Revista Electrónica semestral de } \\
\text { enfermería Global } \\
\text { - Revista Latinoamericana de } \\
\text { Enfermagem } \\
\text { - Journal Nursing Ethics } \\
\text { - Journal of Clinical Nursing }\end{array}$ \\
\hline $\begin{array}{l}\text { Índice de } \\
\text { calidad de } \\
\text { sueño de Pittsburgh } \\
\end{array}$ & PSQI & $\begin{array}{l}\text { Evalúa trastornos del sueño (19 ítems } \\
\text { personales }+ \text { cinco ítems contesta la pareja) }\end{array}$ & Buysse et.al. (1989) & $\begin{array}{l}\text { Artículo } \\
\text { / Revista de Salud Pública }\end{array}$ \\
\hline
\end{tabular}




\section{Revista Electrónica Enfermeria Actual en costa Rica}

\section{www.revenf.ucr.ac.cr}

\begin{tabular}{|c|c|c|c|c|}
\hline $\begin{array}{l}\text { Inventario de síntomas } \\
\text { de } \\
\text { estrés de Lipp }\end{array}$ & ISSL & $\begin{array}{l}\text { Detecta la presencia del estrés en adultos y } \\
\text { la fase que se encuentra: alerta- resistencia - } \\
\text { casi agotamiento- agotamiento. }\end{array}$ & $\begin{array}{l}\text { Lipp y } \\
\text { Guevara } \\
(1994)\end{array}$ & $\begin{array}{l}\text { Artículo }{ }^{43} \\
\text { / Revista enfermagem UERJ }\end{array}$ \\
\hline Prueba de Fagerstrom & FTDN & Evidencia dependencia a la nicotina. & $\begin{array}{l}\text { Fagerstrom KO. } \\
(1978)\end{array}$ & $\begin{array}{l}\text { Artículo }{ }^{24} \\
\text { / Revista Acta Inform Médica }\end{array}$ \\
\hline $\begin{array}{l}\text { Índice de discapacidad } \\
\text { de Oswestry }\end{array}$ & ODI & $\begin{array}{l}\text { Tiene valor predictivo de la codificación del } \\
\text { dolor lumbar }\end{array}$ & O'Brien J.(1980) & $\begin{array}{l}\text { Artículo } 27 \\
\text { / BMC Musculoeskeletal disorders }\end{array}$ \\
\hline $\begin{array}{l}\text { Protocolo de estudio } \\
\text { de análisis de } \\
\text { movimientos }\end{array}$ & $\begin{array}{l}\text { MAGRO- } \\
\text { MSA }\end{array}$ & $\begin{array}{l}\text { Sistema CUELA, medición conectado a la } \\
\text { persona para grabación automatizada de } \\
\text { cargas espinales en actividades profesionales }\end{array}$ & Ellegast R; 1998 & $\begin{array}{l}\text { Medical work assessment in German } \\
\text { hospitals: a study protocol of a } \\
\text { movement sequence analysis } \\
\text { (MAGRO-MSA) } \\
\text { / Journal of occupational Medicine and } \\
\text { Toxicologi }\end{array}$ \\
\hline
\end{tabular}

Fuente: Elaboración propia.

Tabla 3. Universidad de Concepción de Chile. Identificación de las revistas que publican investigaciones en salud laboral, criterios de evaluación revistas por Qualis (46), factor de impacto.

\begin{tabular}{l|c|c|c}
\hline \multicolumn{1}{c|}{ REVISTA } & ESTUDIOS & $\begin{array}{c}\text { CLASIFICACIÓN DE } \\
\text { REVISTA }\end{array}$ & FACTOR DE IMPACTO \\
\hline Revista baiana de enfermagem salvador & 1 & $\mathrm{~B} 2$ & 0.276 \\
\hline Journal Nursing Ethics & 1 & $\mathrm{~A} 1$ & 0.75 \\
\hline Revista Ciencia y Enfermeria & 2 & $\mathrm{~B} 2$ & 1.905 \\
\hline Journal of Nursing Management & 2 & $\mathrm{~A} 1$ & 1.382 \\
\hline Revista Latinoamérica Enfermagem & 7 & $\mathrm{~A} 1$ & 1.214 \\
\hline Journal of Clinical Nursing & 1 & $\mathrm{~A} 1$ & 0.452 \\
\hline Revista da Escola Enfermagem da USP & 4 & $\mathrm{~A} 2$ & 0.40 \\
\hline Revista Española de Salud Pública & 2 & $\mathrm{~B} 2$ & 1.495 \\
\hline Revista enfermagem UERJ & 3 & $\mathrm{~B} 3$ & 0.720 \\
\hline Revista Cuidarte & 1 & $\mathrm{~B}$ & 2.101 \\
\hline Journal of environment Research and Public Health & 2 & $\mathrm{~A} 1$ & 1.482 \\
\hline Journal of occupational Medicine and Toxicology & 2 & $\mathrm{~A} 1$ & 0.96 \\
\hline Journal Occup Environ and medicine & 1 & $\mathrm{~A}$ & 0.365 \\
\hline Revista Acta Inform Medica & 1 & $\mathrm{~A} 1$ & 0.833 \\
\hline Revista Texto \& Contexto Enfermagem & 2 & $\mathrm{~A}$ & 0.789 \\
\hline Revista Electrónica semestral de Enfermería Global & 2 & $\mathrm{C}$ & 2.17 \\
\hline Revista Investigación y educación en Enfermería - Antioquia & 2 & $\mathrm{~B}$ & 0,02 \\
\hline Journal International Archives of Occupational and & 1 & $\mathrm{~A}$ & - \\
\hline Environmental Health & & $\mathrm{C}$ & 3.38 \\
\hline Revista Universidad yalud & 1 & $\mathrm{~B} 3$ & 1.739 \\
\hline Revista chilena de medicina Intensiva & 1 & $\mathrm{~A} 1$ & 2.265 \\
\hline BMC Psiquiatric & 1 & $\mathrm{~A} 1$ & 4.41 \\
\hline BMC Musculoeskeletal disorders & 1 & $\mathrm{~A} 1$ & 1.414 \\
\hline BMC Public Health & 1 & $\mathrm{~A} 1$ & 2.198 \\
\hline Revista Plos one & 1 & $\mathrm{~A} 1$ & $\mathrm{~A} 1$ \\
\hline The european journal of medicine sciencies & 1 & & \\
\hline Neuropsychiatric disease and treatment & 1 & & \\
\hline
\end{tabular}

Fuente: Elaboración propia. 
De la tabla anterior se puede advertir que las publicaciones provienen de 26 revistas evaluadas "A", según criterios Capes de Qualis 2007 46 ; en lo que respecta a nivel de evidencia de los estudios, el $80 \%$ tiene un 4 $(\mathrm{n}=34)$, mientras que ocho revistas tienen 5 y solo 1 tiene 3 , según el método de evaluación utilizados por la Agency for Health Research and Quality (AHRQ) ${ }^{47}$. Con respecto al idioma de las revistas científicas donde fueron publicados estos 45 trabajos, en su mayoría son escritas en portugués $(n=19)$, seguidas por las de idioma inglés $(n=16)$ y 10 , en español. En relación con que aportaron mayor cantidad de trabajos, están las revistas brasileñas (19 estudios), inglesas (ocho), norteamericanas (5), colombianas (4cuatro) como también chilenas y españolas con tres cada una ; finalmente, se identificó un trabajo de Suiza, uno de Bosnia Herzegovina y uno de Alemania. Metodológicamente y según criterios de inclusión se consideró solo estudios cuantitativos.

Luego, se trianguló la lectura crítica de cada uno de los títulos de los artículos con los objetivos de estas investigaciones y los instrumentos utilizados: un $37 \%$ orientó sus escritos a la ansiedad, estrés y acoso que enfrenta la fuerza laboral; un $31 \%$, a la capacidad y condiciones de trabajo; un 19\%, a la detección de riesgo laboral y enfermedades; un 9\%, al tema de autoestima, motivación y empoderamiento y un $2 \%$, a investigar la calidad de vida y sueño de los trabajadores. De esta agrupación de términos, se construyó cinco dimensiones en relación con las diferentes temáticas planteadas en los estudios.

Tabla 4. Universidad de Concepción de Chile. Triangulación de datos, según títulos, objetivos e instrumentos utilizados.

\title{
DIMENSIONES SEGÚN TÍTULO - OBJETIVOS E INSTRUMENTOS UTILIZADOS
}

\section{1) Dimensión psicosocial (Ansiedad- Acoso- Stress)}

\begin{abstract}
Maslach Burnout Inventory > Inventario de síntomas de estrés de Lipp > Inventario de la evaluación de factores estresores subjetivos $>$ Cuestionario relación consumo ansiolítico con el trabajo $>$ Job Content Questionary > Cuestionario de relación de consumo alcohol en el trabajo $>$ Cuestionario de estrés de la conciencia $>$ Cuestionario de percepción de la conciencia > Cuestionario del clima centrado en las persona > Cuestionario de apoyo social $>$ Cuestionario salud general de Goldberg $>$ Cuestionario psicosocial de Copenhague $>$ Escala de síntomas psicosomáticos asociado al stress $>$ Escala de esfuerzo y recompensa $>$ Job Stress Scale $>$ Escala de stress Enfermería $>$ Escala de estrés laboral genérico $>$ Escala de enfrentamiento problema $>$ Scale Stress Nurse $>$ Escala de Ansiedad de Zung $>$ Leyman Inventory Psychological Terrorization
\end{abstract}

\section{2) Dimensión capacidad - trabajo}

Índice de capacidad para el trabajo versión brasileña $>$ Escala de esfuerzo y recompensa $>$ Job content questionnaire $>$ Escala subjetiva de carga mental $>$ Cuestionario condiciones de trabajo $>$ Cuestionario de limitación en el trabajo para el presentismo $>$ Cuestionario de salud y rendimiento del trabajo $>$ Cuestionario costo humano en trabajo $>$ Escala índice de placer y sufrimiento en el trabajo $>$ Escala de afrontamiento ocupacional $>$ Scale Work- Family conflicto y FWC $>$ Scale Impediments in the interactin with patients $>$ Scale barriers to patient oriented work $>$ Stanford Presenteeism Scale $>$ Escala de observación agresión al personal $>$ Escala inestabilidad de trabajo $>$ Scale Patient Oriented control $>$ Scale Patient Orientation $>$ Scale Presenteeism 


\section{3) Dimensión factores de riesgo y detección de la enfermedad}

Cuestionario respiratorio Front Roja $>$ Cuestionario de Boix y Vogel $>$ Prueba de Fagerstrom $>$ Cuestionario Cupid $>$ Cuestionario ocurrencia LBP $>$ Cuestionario Nórdico standarizado >Cuestionario Psicosocial y trabajo Físico $>$ Índice de discapacidad de owestry $>$ Sistema Cuela $>$ Escala de Self- Report Questionnaire-20 $>$ Escala del trabajo y riesgo de Enfermedad $>$ Escala riesgo psicosocial

\section{4) Dimensión autoestima - empoderamiento - motivación}

Escala de autoestima de Rosemberg $>$ Cuestionarios de factores motivacionales $>$ Escala de satisfacción laboral $(\mathrm{RN})>$ Escala de bienestar psicológico $>$ Cuestionario satisfacción laboral $>$ Escala modificada de autoeficacia del comportamiento

\section{5) Dimensión calidad de vida}

Escala de calidad de sueño Pittburg $>$ Escala de calidad de vida de Saint George $>$ Escala abreviada de calidad de vida

Fuente: Elaboración propia.

\section{DISCUSIÓN}

La revisión integrativa permitió no solo el análisis de la información recolectada, sino conocer vacíos del conocimiento y necesidades de futuras investigaciones. De los resultados de esta búsqueda, se advirtió que, en el ámbito internacional, el $65 \%$ de las revistas tiene una clasificación "A", es decir, el mejor concepto en lo respecta a publicaciones en el área de enfermería: a pesar de que también se encuentra revistas del ámbito de la medicina, el mayor porcentaje de estudios presenta un bajo nivel de evidencia; por lo tanto, frente a lo expuesto se advierte un gran desafío para la disciplina que invita a reflexionar en torno a aumentar la complejidad al realizar una investigación.

Se identificó un alto porcentaje de estudios con una mirada desde la atención primaria o en la estrategia de salud en la familia: se observa un número importante de estudios se enfocan en la salud extrahospitalaria $^{24,25,28,48,49,50}$, a diferencia de otros de revisión ${ }^{48}$ en los cuales se detecta escasa publicación desde esta perspectiva. La temática psicosocial es la más abordada en esta revisión -lo cual coincide con otros autores ${ }^{51}$ seguida por los riesgos físicos -entre los que se destaca un gran interés por el estudio del estrés, variable indudablemente traerá repercusiones hacia el trabajador, familia y organización-.

En cuanto al instrumento más utilizado en esta revisión es el Cuestionario de Maslach Burnout Inventory $^{13,22,30,52}$ incluyendo la adaptación japonesa ${ }^{29}$, en el resto de las dimensiones no hubo un instrumento específico que fuera de mayor uso.

Entre las fortalezas de esta revisión, en primer lugar se destaca el aporte al área de salud ocupacional, ya que no se encontró evidencia de investigaciones similares en este ámbito, a pesar que en otras áreas de salud se encontraron resultados afines ${ }^{6,53}$; por otro lado, a partir del análisis de los diversos instrumentos para medir fenómenos o situaciones similares se observa la gran variedad de herramientas encontradas: al respecto, es fundamental que futuros investigadores cuenten con una guía práctica y sencilla para el uso de los instrumentos 
utilizados en el abordaje de salud ocupacional o laboral que permite al investigador escoger el instrumento según su realidad y de acuerdo con el objeto de estudio; por lo tanto, se hace necesario reforzar la preocupación del uso de instrumentos debidamente validados para el control de calidad.

En lo concerniente a las limitaciones de esta revisión, se advierte que no queda claro si, al usar sus instrumentos, los autores se preocuparon por adaptarlos y/o validarlos, a excepción de los artículos ${ }^{16,29,34,35,40}$, los cuales evidenciaron el uso de instrumentos debidamente validados que coincide con resultados de otros estudios $^{54}$ : al vencer esta situación, se conservaría las propiedades psicométricas del instrumento.

\section{CONCLUSIÓN}

Debido a la gran cantidad de artículos encontrados, la contribución de esta revisión, principalmente es recopilar los instrumentos de investigación laboral para orientar a los profesionales de Enfermería al momento que decidan investigar dicha temática. Lo anterior supone un gran reto para la disciplina de Enfermería dado que, por un lado, implica estimular la inquietud de investigar con una mayor ambición de evidencia científica, y por otro lado, invita a estudiar ya no más desde la mirada intrahospitalaria, sino desde lo cotidiano del trabajador, ya que indudablemente desde esa mirada se visibiliza el quehacer de la disciplina en la sociedad, a lo que se suma la necesidad imperiosa de participación política en las esferas gubernamentales, ya que es allí donde se toman las decisiones en lo que respecta a la salud ocupacional.

Declaración de conflictos de intereses: Los autores declaran no tener conflicto de interés de orden personal, comercial, académico, político o financiero en la realización y publicación científica de este documento.

\section{REFERENCIAS BIBLIOGRÁFICAS}

1. Internacional O, Trabajo D. Panorama Laboral 2004. Panor Labor. OIT: Lima; 2004. Disponible en: http://www.ilo.org/wcmsp5/groups/public/---americas/---ro-lima/documents/publication/wcms_187467.pdf

2. WHO. The World Health Report 2006 - working together for health. WHO: Ginebra; 2013. Disponible en: http://www.who.int/whr/2006/en/

3. De Kampala D. Personal de salud para todos. Todos por el personal de salud. OMS: Ginebra; 2008. Disponible en: http://www.who.int/workforcealliance/20090521_Kampala_Sp.pdf

4. OMS. Carta de Bangkok para la promoción de la salud en un mundo globalizado. OMS: Bangkok; 2005. Disponible en: http://www.who.int/healthpromotion/conferences/6gchp/BCHP es.pdf

5. OMS. Foro Mundial sobre Recursos Humanos para la Salud. OMS: Recife; 2013. Disponible en: http://www.who.int/workforcealliance/forum/es/

6. Bauer G. Calidad de vida relacionada con la salud en pediatría . Instrumentos utilizados para su evaluación. Arch Argent Pediatr. 2014; 112(6): 571-6. DOI: http://dx.doi.org/10.5546/aap.2014.571

7. Regina Chitto I. Reflexoes sobre as revistas barsileiras. Porto alegre:UFRGS. 1998; 1(3): 1-10. Disponible en: http://www.seer.ufrgs.br/intexto/article/viewFile/3369/3953

8. Holopainen A, Hakulinen-Viitanen T, Tossavainen K. Systematic review-a method for nursing research. Nurse researcher. 2008; 16: 72-83. DOI: 10.7748/nr2008.10.16.1.72.c6754

9. Pereira SB, Terezinha M, Backes S, Diaz CG, Backes S. Tecnologias apropriadas para o parto e nascimento: 
atribuições do enfermeiro obstetra. Cuidado é fundamental. 2018; 10(2): 29-33. Disponible en: https://dialnet.unirioja.es/ejemplar/503853

10. Sofia Filipe Marote A, Andreia Pinto C, da Rocha Vieira M, do Céu Aguiar Barbiéri-Figueiredo M, Miguel Nunes Pedrosa P. Niños como cuidadores: revisión integrativa. Rev. Latino-Am. Enfermagem. 2012; 20(6). DOI: http://dx.doi.org/10.1590/S0104-11692012000600023

11. Grant MJ, Booth A. A typology of reviews: an analysis of 14 review types and associated methodologies. Heal Inf Libr J. 2009; 26(2): 91-108. DOI: http://doi.wiley.com/10.1111/j.1471-1842.2009.00848.x

12. Prestes FC, Beck CLC, Silva RM Da, Magnago TSB de S, Grecco PBT. Custo Humano No Trabalho Em Enfermagem Em Um Serviço De Hemodiálise. Rev Baiana Enfermagem. 2016; 30(4): 1-10. Disponible en: https://portalseer.ufba.br/index.php/enfermagem/article/view/16440

13. Åhlin J, Ericson-Lidman E, Eriksson S, Norberg A, Strandberg G. Longitudinal relationships between stress of conscience and concepts of importance. Nurs Ethics. 2013; 20(8): 927-42. DOI: https://doi.org/10.1177/0969733013484487

14. Kantek F. Nurses perceptions of motivational factors : a case study in a Turkish university hospital. Journal of Nursing Management. 2015; 23(5): 674-81. DOI: 10.1111/jonm.12195

15. López-Montesinos MJ. Psychosocial study about the consequences of work in hospital nurses as human resource management. Rev. Latino-Am. Enfermagem. 2013; 21(spe): 61-70. DOI: http://dx.doi.org/10.1590/S0104-11692013000700009

16. Campos MC-T de, Marziale MHP, Santos JLF. Adaptação transcultural e validação do World Health Organization Health and Work Performance Questionnaire para enfermeiros brasileiros. Rev da Esc Enferm da USP. 2013; 47(6): 1338-44. DOI: http://dx.doi.org/10.1590/S0080-623420130000600013

17. de Oliveira EB, Araujo PMB, Maia MPQ, de Cabral JL, de Brito DM, de Figueredo EP. Estresse ocupacional e consumo de ansiolíticos por trabalhadores de enfermagem. Rev Enferm. 2014; 22(5): 615-21. Disponible en: http://www.redalyc.org/pdf/408/40849134018.pdf

18. Salud UY, Zapata-Herrera M, Zapata-Gómez NE. Working conditions of nurses and the forms of contracting in Medellín, Colombia. Univ. Salud. 2015; 17(2): 212-23. Disponible en:

http://www.scielo.org.co/scielo.php?script=sci arttext\&pid=S0124-71072015000200007

19. Umann J, Guido L de A, da Silva RM. Stress, coping and presenteeism in nurses assisting critical and potentially critical patients. Rev da Esc Enferm. 2014; 48(5): 887-94. DOI: http://dx.doi.org/10.1590/S00806234201400005000016

20. Barbosa de Oliveira E, Greyciele da Silva Matos I. Padrões de uso de alcool Por Trabalhadores de Enfermagem e a associação com o trabalho. Rev enferm. 2013; 21(2): 729-35729. Disponible en: http://www.facenf.uerj.br/v21esp2/v21e2a06.pdf

21. Rieder K, Faedi J, Elke R. Work stressors and resources in a Swiss orthopaedic clinic and their relationship with employees' health and the patient orientation of employees. Swiss Med Wkly. 2012; 142: 1-10. DOI: 10.4414/smw.2012.13532

22. Portero de la Cruz S, Vaquero Abellán M. Professional burnout, stress and job satisfaction of nursing staff at a university hospital. Rev. Latino-Am. Enfermagem. 2015; 23(3): 543-52. DOI:

http://dx.doi.org/10.1590/0104-1169.0284.2586

23. Feitosa Beleza CM, Teles de Oliveira Gouveia M, Do Carmo Cruz Robazzi ML, Dias Torres CR, Viana De Azevedo GA. Riscos Ocupacionais E Problemas De Saúde Percebidos Por Trabalhadores De Enfermagem Em Unidade Hospitalar. Cienc y enfermería. 2013; 19(3): 63-71. DOI: http://dx.doi.org/10.4067/S0717$\underline{95532013000300008}$ 
24. Koulouri A, Gourgoulianis K, Hatzoglou C, Roupa Z. Telemetric detection of chronic obstructive pulmonary disease and investigation of quality of life for people working in shipbuilding industry. Acta Inform Med. 2014; 22(5): 315-9. DOI: 10.5455/aim.2014.22.315-319

25. Rotenberg L, Silva-Costa A, Härter Griep R. Mental health and poor recovery in female nursing workers: a contribution to the study of gender inequities. Rev Panam Salud Publica. 2014; 35(3): 179-85. Disponible en: https://pdfs.semanticscholar.org/7779/02d14478a2d8b76e4aeb6b8c4d179af750bf.pdf?.ga=2.252493322.245 676518.1560461717-65878861.1560461717

26. Kawaguchi M, Matsudaira K, Sawada T, Koga T, Ishizuka A, Isomura T, et al. Assessment of potential risk factors for new onset disabling low back pain in Japanese workers: Findings from the CUPID (cultural and psychosocial influences on disability) study. BMC Musculoskelet Disord. 2017; 18(1): 1-8. DOI:

10.1186/s12891-017-1686-y

27. Erick PN, Smith DR. Low back pain among school teachers in Botswana, prevalence and risk factors. BMC Musculoskelet Disord. 2014; 15: 359. DOI: 10.1186/1471-2474-15-359

28. Zhang X, Chen G, Xu F, Zhou K, Zhuang G. Health-related quality of life and associated factors of frontline railway workers: A cross-sectional survey in the Ankang Area, Shaanxi Province, China. Int J Environ Res Public Health. 2016; 13(12). DOI: 10.3390/ijerph13121192

29. Sugawara N, Danjo K, Furukori H, Sato Y, Tomita T, Fujii A, et al. Work-family conflict as a mediator between occupational stress and psychological health among mental health nurses in Japan. Neuropsychiatr Dis Treat. 2017; 13: 779-84. DOI: 10.2147/NDT.S127053

30. Wu H, Liu L, Wang Y, Gao F, Zhao X, Wang L. Factors associated with burnout among Chinese hospital doctors: a cross-sectional study. BMC Public Health. 2013; 13(1): 786. DOI: https://doi.org/10.1186/14712458-13-786

31. Prestes FC, Beck CLC, Magnago TSB de S, da Silva RM. Pleasure-suffering indicators of nursing work in a hemodialysis nursing service. Rev da Esc Enferm. 2015; 49(3): 465-72. DOI: http://dx.doi.org/10.1590/S0080-623420150000300015

32. Sparks AM. Psychological empowerment and job satisfaction between Baby Boomer and Generation X nurses. J Nurs Manag. 2012; 20(4): 451-60. DOI: 10.1111/j.1365-2834.2011.01282.x

33. Palhares V de C, Corrente JE, Matsubara BB. Association between sleep quality and quality of life in nursing professionals working rotating shifts. Rev Saude Publica. 2014; 48(4): 594-601. DOI: http://dx.doi.org/10.1590/S0034-8910.2014048004939

34. Solange T, De B, Magnago S, Cláudia A, De Lima S, Prochnow A, et al. Intensidad del dolor musculoesquelético y la (in)capacidad para el trabajo en la enfermería. Rev. Latino-Am. Enfermagem. 2012; 20(6). DOI: http://dx.doi.org/10.1590/S0104-11692012000600015

35. Petri Tavares J, Colomé Beck CL, Bosi de Souza Magnano TS, Ruviaro Zanini R, Lautert L. Disturbios psíquicos menores en enfermeros docentes universitarios. Rev. Latino-Am. Enfermagem. 2012; 20(1): 1-8. Disponible en: http://www.scielo.br/pdf/rlae/v20n1/pt 23.pdf

36. Terra FS, Marziale MHP, Robazzi MLCC. Avaliação da autoestima em docentes de enfermagem de universidades publica e privada. Rev. Lat-Am. Enfermagem. 2013; 21: 1-8. DOI:

http://dx.doi.org/10.1590/S0104-11692013000700010

37. Vazquez P, Paravic T, Burgos M, Barriga O. Validation of subjective mental Workload Scale in academic staff. Ciencia y Enfermería. 2014; 20(2): 73-82. Disponible en: https://www.researchgate.net/publication/287235053_Validation_of_subjective_mental_workload_scale_in academics_staff 
38. Prochnow A, Beatriz S, Lima S De. Capacidad para el trabajo en enfermería : relación con demandas psicológicas y control sobre el trabajo. Rev. Latino-Am. Enfermagem. 2013; 21(6): 1298-305. DOI: 10.1590/0104-1169.3072.2367

39. Pereira SDS, Araujo C, Teixeira B, Reisdorfer E, Vieira MV. The relationship between occupational stressors and coping strategies in nursing technicians. Texto contexto enferm. 2016; 25(4): 1-8. DOI: http://dx.doi.org/10.1590/0104-07072016002920014

40. Harling M, Schablon A, Peters C, Nienhaus A. Predictive values and other quality criteria of the German version of the Nurse-Work Instability Scale (Nurse-WIS) - Follow-up survey findings of a prospective study of a cohort of geriatric care workers. J Occup Med Toxicol. 2014; 9(1): 1-12. DOI: 10.1186/s12995-0140030-9

41. Hao J, Wang J, Liu L, Wu W, Wu H. Perceived organizational support impacts on the associations of workfamily conflict or family-work conflict with depressive symptoms among Chinese doctors. Int J Environ Res Public Health. 2016; 13(3): 1-13. DOI: 10.3390/ijerph13030326

42. Fontes KB, Santana RG, Pelloso SM, Dalva M, Carvalho B. Factores asociados al acoso moral en el ambiente laboral del enfermero. Rev. Latino-Am. Enfermagem. 2013; 21(3). DOI: http://dx.doi.org/10.1590/S0104-11692013000300015

43. Kestenberg CCF, Felipe ICV, Rossone F de O, Delphim LM, Teotonio MC. O estresse do trabalhador de enfermagem: Estudo em diferentes unidades de um hospital universitário. Rev Enferm. 2015; 23(1): 45-51. DOI: http://dx.doi.org/10.12957/reuerj.2015.11487

44. Muñoz, M., Molina P. Síndrome de Burnout en el Equipo de Salud de una Unidad de Cuidados Intensivos, Concepción. Rev Chil Med Intensiva. 2013; 28(1): 13-8. Disponible en: https://www.medicinaintensiva.cl/revistaweb/revistas/indice/2013-1/pdf/3.pdf

45. Ohlendorf D, Schwarzer M, Rey J, Hermanns I, Nienhaus A, Ellegast R, et al. Medical work assessment in German hospitals: A study protocol of a movement sequence analysis (MAGRO-MSA). J Occup Med Toxicol. 2015; 10(1): 1-8. DOI: 10.1186/s12995-014-0040-7

46. Erdmann AL, Helena M, Marziale P, Da Luz M, Pedreira G, Carlos F, et al. La evaluación de los periódicos científicos Qualis y la producción brasileña de artículos del área de Enfermería. Rev. Latino-Am. Enfermagem. 2009; 17(3). DOI: http://dx.doi.org/10.1590/S0104-11692009000300019

47. Caspi CE, Dennerlein JT, Kenwood C, Stoddard AM, Hopcia K, Hashimoto D, et al. Results of a Pilot Intervention to Improve Health and Safety for Health Care Workers. J Occup Environ Med. 2013; 55(12): 1449-55. DOI: 10.1097/JOM.0b013e3182a7e65a

48. Nogueira C, Nobrega L, Aline G, Sostenes S, Soares F MR. The scientific production about occupational healt of nursing. Rev Pesqui Cuid e Fundam. 2013; 5(5): 52-60. DOI: 10.9789/2175-5361.2013v5n5esp52

49. Loiola Moura A, Margarete Dos Reis L, Terezinha M, Vannuchi O, Do M, Haddad CL, et al. Capacidade para o trabalho de funcionários da prefeitura de um campus universitário público. Artig Orig Rev Eletr Enf. 2013; 15(1): 130-7. DOI: http://dx.doi.org/10.5216/ree.v15i1.13574

50. Adol Worm F, Aparecida Oliveira Pinto M, Schiavenato D, Amora Ascari R, De Lima Trindade L, Martins da Silva O. Risco de adoecimento dos profissionais de enfermagem no trabalho em atendimento móvel de urgência. Rev Cuid. 2016; 7(2): 1288-96. DOI: https://doi.org/10.15649/cuidarte.v7i2.329

51. Chaparro C, Cocunubo L, Pulido N. Estado del arte de las investigaciones sobre salud ocupacional en las escuelas de psicología y enfermería de la Universidad Pedagógica y Tecnológica de Colombia entre 1983 y 2010. Rev Fac Nac Salud Pública. 2011; 30(2): 131-40. Disponible en: http://www.scielo.org.co/pdf/rfnsp/v30n2/v30n2a02.pdf 
52. Hensel JM, Lunsky Y, Dewa CS. The mediating effect of severity of client aggression on burnout between hospital inpatient and community residential staff who support adults with intellectual disabilities. J Clin Nurs. 2014; 23(9-10): 1332-41. DOI: 10.1111/jocn.12387

53. Manterola C, Urrutia S, Otzen T. Calidad de vida relacionada con salud: instrumentos de medición para valoracion de resultados en cirugia digestiva alta. Rev Chil Cir. 2014; 66(3): 274-82. DOI: http://dx.doi.org/10.4067/S0718-40262014000300016

54. Te T, Sa FJ. Instrumentos especificos de medida de la calidad de vida relacionada con la salud en la diabetes en la mellitus tipo 2 disponibles en España. Med Clin. 2010; 135(14): 658-64. DOI: 10.1016/j.medcli.2009.04.029 